\title{
Popularity Prediction of Videos in IPTV Systems
}

\author{
Reyhaneh Ghavami, Alireza Abdollahpouri, Zhila Bahrami, Parham Moradi \\ Department of Computer Engineering and Information Technology \\ University of Kurdistan, Sanandaj, Iran \\ Emails: reyhan.ghavamy.191@gmail.com,abdollahpour@uok.ac.ir,bahrami.zhila@gmail.com,p.moradi@uok.ac.ir
}

\begin{abstract}
Video on demand (VoD) service in IPTV is a bandwidth-hungry application. It has been argued that the distribution of popularity of videos can be well measured using a Zipf-like distribution in which top $10 \%$ of the videos account for nearly $90 \%$ of requests. In this article, we propose a neural network based method to predict the popularity of videos in an IPTV system. The popularity prediction can be used by service providers for video placement in content delivery systems or hierarchical servers and hence it can lead to bandwidth save. Simulation-based performance evaluation of our proposed method confirms a significant accuracy in the prediction of the popularity of the videos.
\end{abstract}

Keywords: IPTV,Video Popularity, Artificial Neural Network.

\section{INTRODUCTION}

We are currently witnessing a paradigm shift from push-based media broadcasting to pull-based media streaming. Services like pay-per-view and video on demand $(\mathrm{VoD})$ are becoming more and more common and are changing the way we look at multimedia. The growth of multimedia applications has been explosive in the last decade. This rapid growth is partially due to the advances in media encoding and compression techniques (e.g., H.264/AVC) and partially because of the enormous improvement of networking technologies. Internet Protocol Television (IPTV) describes a mechanism for transporting streams of video content encapsulated in IP packets over the network using networking protocols of the TCP/IP protocol stack. It provides a so called "walled garden" architecture (closed or exclusive set of services provided for users) to guarantee necessary QoE for subscribers [8].

IPTV is one of the fastest growing services, such that, the number of worldwide IPTV subscribers is expected to exceed 70 million by 2015 [1]. Commercial deployments of IPTV services by Telcos around the world continue to increase [2], [3], [4].

IPTV services can be divided into two broad classes: live streaming applications and on-demand applications. Live TV applications are delivered as the data is being captured and encoded. In this approach, there are strict timing requirements for the delivery of TV channels. In contrast to the live TV streaming, in video on demand applications, stored media files are delivered to the client at the time when they are requested. On-demand videos can be delivered using three techniques: download, progressive download and streaming [8].

In $\mathrm{VoD}$ service, the popularity of different videos is not equal. It has been argued that the distribution of popularity of videos can be well measured using a Zipflike distribution in which top $10 \%$ of the videos account for nearly $90 \%$ of viewers (see Fig. 1). Therefore, most of the videos are unpopular or rarely watched. Service providers can take advantage of this property to design and implement a suitable caching or content delivery systems where popular videos will be available close to the customer while a rarely watched video may only be available further up in the hierarchically structured network.

Prediction of the video popularity can be accomplished by different methods. Artificial neural network can be a useful tool for this purpose.

Meanwhile, to increase the performance of IPTV systems, the higher popularity items can be recommended to users. In [5] an advanced IPTV services personalization model was proposed for context-aware content recommendation by S. Song et. al. Their work has high accuracy, because they considered context information in their recommendation system. M. Krstić et. al in [6] used context information as input of neural network and clustered videos to like and dislike video groups. Jun et. al, in [7] used historical information of one week in a dataset obtained from monitoring the real VoD system and proposed a neural network for estimate popularity of videos. They considered equal weights for all the days of the week.

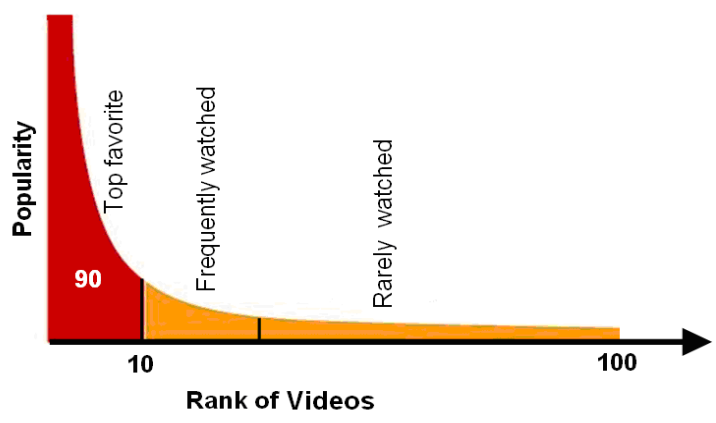

Fig. 1: Popularity distribution of videos 
In this paper, we try to predict the popularity of videos in an IPTV system using a 3-layer neural network. To some extent, our work is similar to [7]; but we use different weights in our method and less neurons, as well.

The rest of the paper is organized as follows. In Section II, some background information regarding IPTV, video popularity measurement and neural networks is presented. Section III introduces our proposed method as well as required assumptions. Experimental results and performance evaluation is given in Section IV. Finally, we conclude the paper in Section V.

\section{BACKGROUND}

\section{A. IPTV}

IPTV describes a mechanism for transporting TV streams encapsulated in IP packets using networking protocols and tries to offer more interactivity and more control over the content. Fig. 2 depicts a typical IPTV service network architecture which provides triple play services (i.e., voice, video and data) via xDSL access network. An IPTV system mainly consists of five different parts as shown in the figure [8].

\section{IPTV Head-end:}

This part of the IPTV system is responsible for acquiring, processing, encoding, and managing video content. Video content is typically compressed using either MPEG-2 or MPEG-4 codec and then it is sent using MPEG transport stream (MPEG-TS) packets which are delivered by means of IP multicast or unicast. A typical IP packet for transporting MPEG video contains seven 188-byte MPEG-TS packets. The head-end manages access to on-demand videos as well.

\section{Core Network:}

The core network is the central portion of an IPTV system. It primarily provides interconnection between several metro networks. In order to reduce latency between the clients and the streaming servers and therefore improve the experienced QoS, core networks use fibre optic links. Meanwhile, multicast enabled routers are employed to deliver TV channels and Videos to the distribution network. Traffic engineering techniques using MPLS can also help the subscribers to experience a better video quality.

\section{Distribution Network (Metro backbone):}

The distribution network typically serves a region or a metropolitan area. It inserts local content such as local TV channels or commercial advertisements into the IPTV streams and provides on-demand video services to the clients located in its region. Typical equipment in this part consists of the encoders for local TV channels, local advertisement inserters and video servers to stream ondemand video services.

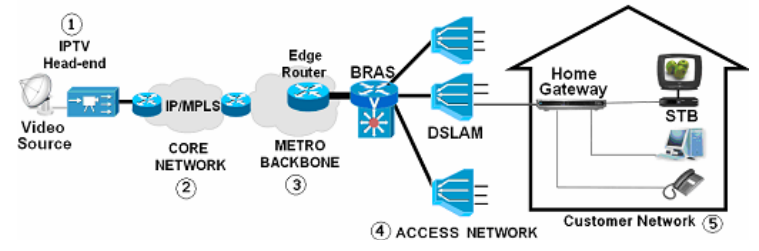

Fig 2: Structure of an IPTV System

\section{Access Network:}

The access network is an essential part of the IPTV structure and acts as a border between service provider and subscriber. It provides last mile access for IPTV subscribers and can be based on wired technologies (e.g., xDSL, FTTx) or wireless technologies. In Fig. 2, an access network based on xDSL technology is shown.

\section{Customer Network:}

The customer network provides TV, IP phone and Internet services to subscribers. It connects the home computer(s), the IPTV Set-Top Box (STB) and IP phone device to a broadband service which is provided by the access network via a home gateway. This network may also support Voice over IP (VoIP) services.

Compared with other Internet services, e.g., online surfing, gaming and VoIP, IPTV service not only consumes higher bandwidth, but also requires a higher degree of end-to-end Quality of Service (QoS) guarantees throughout the delivery path. Thus, the user QoE is sensitive to packet loss, delay and jitter. Techniques such as Scalable Video Coding (SVC) open opportunities for adapting streaming of IPTV channels to improve QoS.

In this paper, our focus is on the VoD service and therefore, popularity modelling and measuring of videos will be introduced in the following subsection in some more detail.

\section{B. Video Popularity Definition and Modeling}

Popularity is an important factor in estimating the request probability of an item. Identifying the video popularity is important for traffic engineering and for design and evaluation of media distribution systems. Thus, it is highly desirable to have a clear understanding of media access patterns for both media system designers and network administrators. In VoD service, the popularity can be measured as the number of requests during a time interval.

In [7] the authors used the following equation to quantify the popularity of videos in a VoD system:

$$
\operatorname{req}^{\prime}(i)_{t}=\log \left(\operatorname{req}(i)_{t}+1\right)
$$

where, $i$ is number of item and $t$ is the day. Then, the popularity is calculated as follows:

$$
\operatorname{pop}(i)_{t}=\frac{\operatorname{req}^{\prime}(i)_{t}}{\sum_{i=1}^{N} r e q^{\prime}(i)_{t} / N}
$$


Probability distribution of video can be modelled quite realistically using a Zipf-like distribution with the following formula $\left(P_{i}\right.$ is the watching probability of $i$ thranked video):

$$
p_{i}=\frac{\Omega}{i^{\alpha}}
$$

where,

$$
\Omega=\left(\sum_{j=1}^{C} \frac{1}{j^{\alpha}}\right)^{-1}, 0<\alpha \leq 1
$$

Here, $\alpha$ is the shaping parameter, $\Omega$ is the scaling parameter, which can be decided by $\alpha$, and $\mathrm{C}$ is the number of videos.

\section{Artificial Neural Network}

Neural network (NN) is a mathematical and computational model that is inspired by the structure of functional aspects of biological neural network [9]. A neural network consists of an interconnected group of artificial neurons, and processes information using a connectionist approach to computation. Modern neural networks are usually used as non-linear statistical data modeling tools. They are usually used to model complex relationships between inputs and outputs or to find patterns in data. It widely used in prediction environments. At first the network is trained using data train; then the proper weights between neurons are calculated. After that function can be estimated.

Fig. 3 shows a typical 3-layer neural network which consists of input layer, hidden layer and output layer.

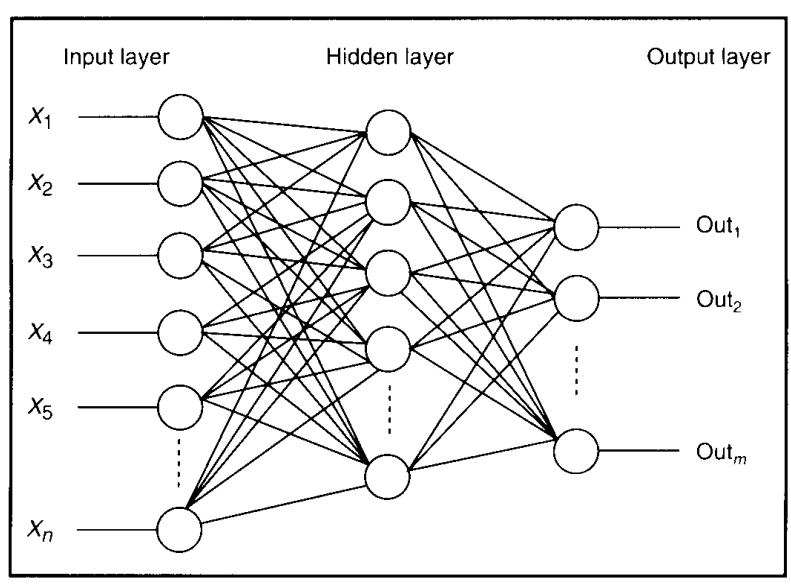

Fig 3: Structure of neural network

\section{PROPOSED METHOD}

In this section, our proposed popularity prediction is discussed. The popularity of the video is estimated based on the historical information of it. By historical information, we mean the total number of requests by the users for videos in previous days. The information of the video requests for one month time interval is derived from the dataset and the popularities of the videos are calculated using formula (1). It's worth noting that all the previous days are not equally important in calculating the popularity for the next day. In other words, we use different weights for each day. A 3-layer neural network with eight inputs in the first layer according to Fig. 4 is used for this purpose. We consider the information of 7 last days as 7 inputs of the neural network and the sum of information of 30 days as the last input. Also, according to what we discussed previously about the different importance of the days, we assign different weights to each input as follows. The weight of yesterday is 0.3 and the weight of two days ago is 0.2 , and accordingly the value of 0.1 is considered for 3,4 and 5 day ago, and the value of 0.066 is assigned to 6,7 and sum up 30 days ago. Equation (5) depicts the input vector of neural network.

$$
\begin{aligned}
& \text { Inp }=\left(0.3 * \operatorname{pop}(i)_{t-1}+0.2 * \operatorname{pop}(i)_{t-2}+0.1 * \operatorname{pop}(i)_{t-3}+\right. \\
& 0.1 * \operatorname{pop}(i)_{t-4}+0.1 * \operatorname{pop}(i)_{t-5}+0.066 * \operatorname{pop}(i)_{t-6}+ \\
& \left.0.066 * \operatorname{pop}(i)_{t-7}+0.66 * \sum_{j=1}^{30} \operatorname{pop}(i)_{t-j}\right)
\end{aligned}
$$

where Inp is the input vector of the neural network (with the size of 8).

Fig. 4 illustrates the structure of $\mathrm{NN}$ we used. As mentioned earlier, it has three layers. The first layer consists of eight neurons to get historical information. Hidden layer has three neurons and output layer has one neuron that determines the popularity of video in the range of $[0,1]$. Activation functions in all layers are set as Sigmoid function in (6).

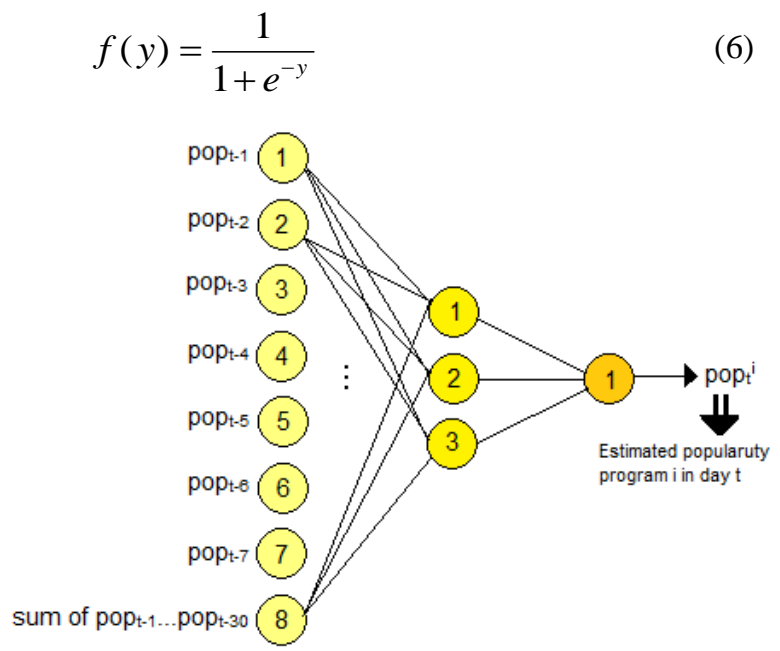

Fig 4: Structure of proposed neural network

We use Back Propagation (BP) training algorithm, for training our neural network. The pseudo code of our proposed method is given in Fig 5. At the first step, historical information of 1 month ago is read from the dataset and the popularity of each video is calculated in the second step. In step 3, input vector of the neural network is created based on popularities. Then, our neural network is trained and output from neural network is given. In the next step, videos sorted by descending 
order of popularity. In the last step, some hot programs according to their rank and capacity of system are selected.

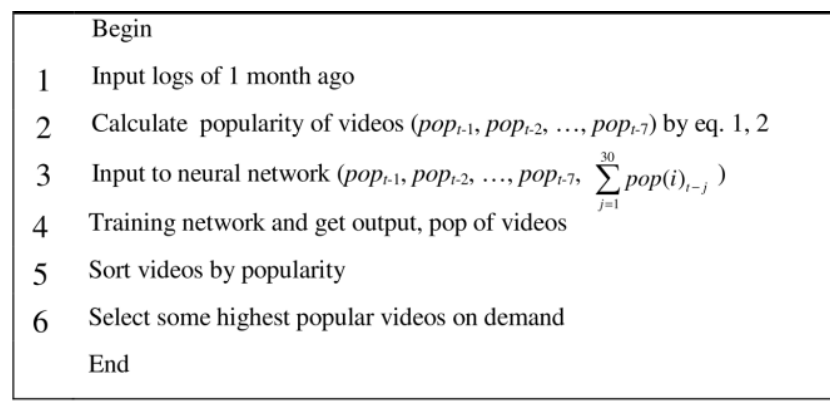

Fig 5: Pseudo code of proposed algorithm

\section{EXPERIMENTAL RESULTS}

In this section, we present our experimental results based on prediction of our neural network and compare it with another NN-based predictor algorithm [7]. Another strategy that can be used is LRU (Last Recently Used), which considers data of only one day ago. So we compare our method with the above two strategies.

Unfortunately, the dataset of [7] is not publicly accessible (maybe for security reasons). Therefore, we tried to produce a (so called) artificial dataset. As mentioned previously, video popularity has a Zipf-like distribution. Taking advantage of this property, we made a dataset using equations 3, 4. Our dataset contains 100000 records of about 14000 videos which have been requested during 1 month time interval.

To evaluate the performance of our method and in order to compare it with other methods, we use AR metric which is defined as follows:

\section{$\mathrm{AR}=\frac{\text { Number of videos that estimated correctly }}{\text { Total number of videos }}$}

The number of videos is varied from 3000 to 14000 (with the step of 1000), and we train network with these videos.

To study the performance of our method we use our artificial dataset and compare it to other NN-based predictor methods. The results are listed in Table 1 and are shown in Fig. 6. The horizontal axis shows amount of videos, and the vertical axis shows accuracy of different methods. In this figure blue curve illustrates accuracy of our methods, red curve illustrate accuracy of NN-based prediction and green curve depicts accuracy of LRU method.

It can be deducted from the results that with the increment of number of videos the accuracy increases and the curve related to our method remains above the curves of the similar methods which indicated the better performance of the proposed method. For example, when the number of videos is 3000 , the accuracy of our approach is above 0.88 . While the AR for LRU and other
NN-based method is 0.79 and 0.80 , respectively. Similarly, for the number of videos raised to 14000 , the accuracy gain is 0.94 while it is 0.85 in LRU and 0.89 in NN-based prediction.

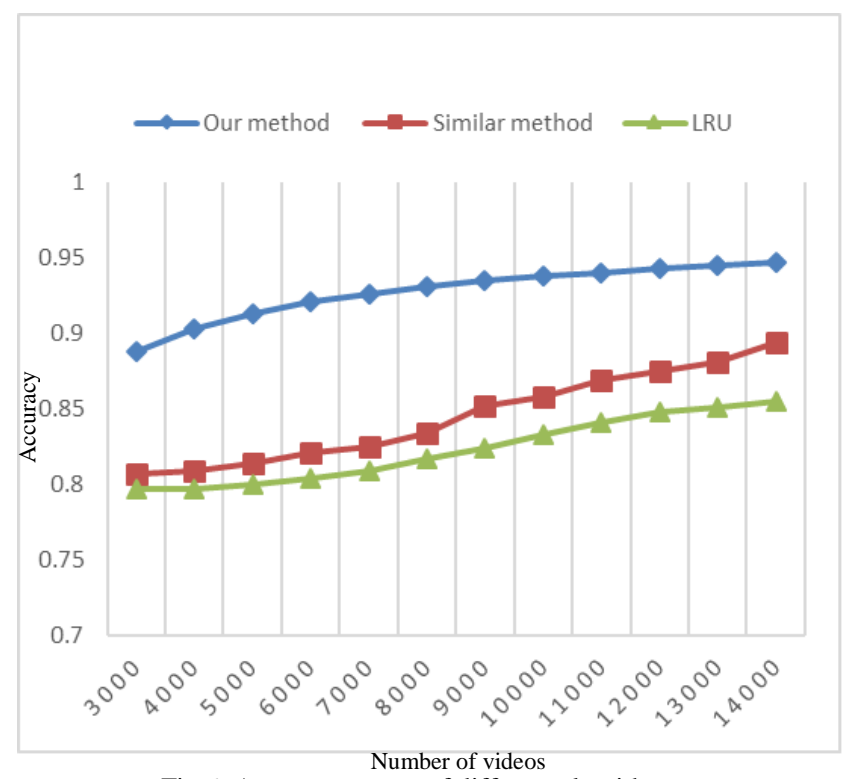

Fig 6: Accuracy curves of different algorithms

Table 1: Accuracy of different algorithms

\begin{tabular}{|c|c|c|c|}
\hline $\begin{array}{c}\text { Number } \\
\text { of } \\
\text { videos }\end{array}$ & NN-proposed & $\begin{array}{l}\text { NN-based } \\
\text { predictor }\end{array}$ & LRU \\
\hline 3000 & 0.888391904 & 0.8072 & 0.7965 \\
\hline 4000 & 0.902758501 & 0.8087 & 0.7968 \\
\hline 5000 & 0.912988597 & 0.8136 & 0.8001 \\
\hline 6000 & 0.920662067 & 0.8214 & 0.8043 \\
\hline 7000 & 0.926281317 & 0.8247 & 0.809 \\
\hline 8000 & 0.930832352 & 0.8344 & 0.8165 \\
\hline 9000 & 0.934698103 & 0.8522 & 0.8242 \\
\hline 10000 & 0.937771695 & 0.8578 & 0.833 \\
\hline 11000 & 0.940524267 & 0.8692 & 0.8411 \\
\hline 12000 & 0.943186921 & 0.8755 & 0.8476 \\
\hline 13000 & 0.94550896 & 0.8812 & 0.8513 \\
\hline 14000 & 0.94738286 & 0.8938 & 0.855 \\
\hline
\end{tabular}

In our method, the historical information of one month is used and the neural network we use has eight inputs, including 7 past days and sum-up of one month ago. NN makes decision based on this information. Since we use more information in the network, our method has more accuracy than others.

In LRU method, only information of the past day is used. So our approach is better than LRU in terms of accuracy. Also, in another NN-Based prediction, the historical information of all the days of the last week has same importance with equal weights. It is worth mentioning that in the method of [7] the authors use 6 neurons in hidden layer to train the network, while we use only 3 neurons in the hidden layer. The reduction of hidden neurons causes shorter network training time. In addition, since different days have different effects on popularity of videos, assigning different weights seems quite reasonable and leads to higher accuracy in 
popularity prediction of videos. The output of our method is fuzzy and is in the range of $[0,1]$. While the network output of NN-based method in [7] has only two values of 0 and 1 . Therefore, sorting of videos is not very useful in that method. But in our method, since the output is in the range of $[0,1]$, sorting make sense and is useful.

\section{CONCLUSION}

In this paper, a method to estimate the popularity of videos in IPTV systems based on artificial neural network was proposed. We used historical information (last 30 days) of requested items. Popularity of videos in every day, are calculated based on number of requests. These popularities are considered as input vector of a 3layer neural network which is trained by BP algorithm. Output of the network is the popularity estimation of the program in the range of $[0,1]$. Since all the previous days are not equally important in calculating the popularity for the next day, we proposed a weighted sum for the popularity estimation in which for example, the number of requests of yesterday is more important than of two days ago. To evaluate the performance of our method, we used AR metric and compared our method with LRU and NN-based predictor. According to experimental result, our proposed method can achieve about 0.1 more accuracy.

As a potential future work, in addition of getting historical information, we can get context information for videos and requests. Also, when a real dataset is accessible, the accuracy of the method can be tested again. In this paper, we focused on VoD services in IPTV systems. However, one can use this approach in live TV services.

\section{References}

[1] IMS Research. A Global Market Analysis, 2011 Edition.

[2] http://www.att.com/u-verse (last visited: Feb. 2014)

[3] http://www.Telekom.de/Entertain (last visited: Feb. 2014)

[4] http://www.telefonica.com (last visited: Feb. 2014)

[5] S. Songbo, H. Moustafa, and H. Afifi, "Advanced IPTV services personalization through context-aware content recommendation" Multimedia, IEEE Transactions on 14.6 (2012): pp. 1528-1537.

[6] K. Marko, and M. Bjelica, "Context-aware personalized program guide based on neural network," Consumer Electronics, IEEE Transactions on 58.4 (2012): pp. 1301-1306.

[7] J. Li, S. Hong and S. Xia, "Neural Network Based Popularity Prediction For IPTV System" J. of Networks, V.7 No.12 Dec. 2012.

[8] A. Abdollahpouri, B. E. Wolfinger, J. Lai, and C. Vinti, "Elaboration and formal description of IPTV user models and their application to IPTV system analysis," in Proceedings of MMBnet 2011 Workshop, Hamburg, Germany, Sept. 2011.

[9] Hagan, Martin T., Howard B. Demuth, and Mark H. Beale. Neural network design. Boston: Pws Pub., 1996. 